\title{
Incidência de lesões da rampa meniscal nas reconstruções do ligamento cruzado anterior*
}

\section{Incidence of Meniscal Ramp Lesions in Anterior Cruciate Ligament Reconstructions}

\author{
Felipe Galvão Abreu1수 Sérgio Marinho de Gusmão Canuto ${ }^{20}$ Maira Macedo de Gusmão Canuto ${ }^{20}$ \\ Eduardo Federighi Chagas ${ }^{3(1)}$ Tereza Lais Menegucci Zutin ${ }^{4(1)}$ Vitor Barion Castro de Pádua4(0)
}

${ }^{1}$ Clínica Sportsmed, São José do Rio Preto, SP, Brasil

2 Ortoclínica Hospital de Ortopedia, Maceió, AL, Brasil

${ }^{3}$ Departamento de Educação Física da Universidade de Marília, Marília, SP, Brasil

${ }^{4}$ Departamento de Ortopedia e Traumatologia, Universidade de Marília, Marília, SP, Brasil

Rev Bras Ortop 2022;57(3):422-428.
Endereço para correspondência Felipe Galvão Abreu, MD, Av. Juscelino Kubitschek de Oliveira, 5.000, Sala 509, Complexo Iguatemi, São José do Rio Preto, SP, 15093-270, Brasil

(e-mail: dr.felipegalvao@hotmail.com).

\section{Resumo \\ Palavras-chave \\ - artroscopia \\ - epidemiologia \\ - joelho \\ - sutura \\ Objetivo Avaliar a incidência e o perfil epidemiológico das lesões da rampa meniscal nos pacientes submetidos a cirurgia de reconstrução do ligamento cruzado anterior (LCA), e determinar os fatores de risco relacionados. \\ Métodos Foram analisados retrospectivamente 824 pacientes submetidos a cirurgia de reconstrução do LCA. Os pacientes que apresentaram instabilidade meniscal medial foram submetidos a avaliação do compartimento posteromedial do joelho. Em caso de lesão, o reparo cirúrgico foi realizado. Potenciais fatores de risco associados às lesões foram analisados. Resultados A incidência geral de lesões da rampa na população estudada foi de $10,6 \%$ (87 lesões em 824 pacientes). A análise multivariada pelo teste do Qui-quadrado demonstrou que a presença de lesões da rampa meniscal foi significativamente associada aos seguintes fatores de risco: lateralidade direita e lesões crônicas. Sexo, idade e atividade esportiva não foram estatisticamente significantes. O futebol foi a causa mais frequente de lesões da rampa relacionadas ao esporte, com $78,2 \%$ dos casos. No entanto, não se mostrou ser um fator de risco. De 2014 a 2019, a incidência anual variou de $4,0 \%$ a $20,6 \%$. \\ Conclusão A incidência das lesões da rampa meniscal foi de $10,6 \%$ nas cirurgias de reconstrução do LCA, sendo mais frequente em pacientes com lesões crônicas. A incidência anual foi crescente, e variou de 4,0\%, em 2014, a 20,6\%, em 2019.}

Trabalho desenvolvido na Clínica Sportsmed, São José do Rio Preto, SP; no Departamento de Ortopedia e Traumatologia da Universidade de Marília, Marília, SP; e na Ortoclínica Hospital de Ortopedia, Maceió, AL, Brasil.

recebido

04 de Dezembro de 2020

aceito

02 de Junho de 2021

Publicado on-line

de Janeiro 20, 2022
DOI https://doi.org/ $10.1055 / \mathrm{s}-0041-1735942$ ISSN 0102-3616. (c) 2022. Sociedade Brasileira de Ortopedia e Traumatologia. All rights reserved.

This is an open access article published by Thieme under the terms of the Creative Commons Attribution-NonDerivative-NonCommercial-License, permitting copying and reproduction so long as the original work is given appropriate credit. Contents may not be used for commercial purposes, or adapted, remixed, transformed or built upon. (https://creativecommons.org/ licenses/by-nc-nd/4.0/)

Thieme Revinter Publicações Ltda., Rua do Matoso 170, Rio de Janeiro, RJ, CEP 20270-135, Brazil 
Abstract
Keywords
- arthroscopy
- epidemiology
- knee
- suture

Objective To evaluate the incidence and epidemiological profile of meniscal ramp lesions in patients undergoing anterior cruciate ligament $(\mathrm{ACL})$ reconstruction surgery, and to determine the related risk factors.

Methods In total, 824 patients undergoing ACL reconstruction surgery were retrospectively analyzed. Patients who presented medial meniscal instability were submitted to evaluation of the posteromedial compartment of the knee. In case of injury, surgical repair was performed. Potential risk factors associated with the lesions were analyzed.

Results The overall incidence of ramp lesions in the population studied was of $10.6 \%$ (87 lesions in 824 patients). The multivariate analysis through the Chi-squared test showed that the presence of meniscal ramp lesions was significantly associated with the following risk factors: right laterality and chronic lesions. Gender, age and sports activity were not statistically significant. Soccer was the most frequent cause of ramp injuries related to sport, with $78.2 \%$ of the cases. However, it was not shown to be a risk factor. The annual incidence from 2014 to 2019 ranged from $4.0 \%$ to $20.6 \%$.

Conclusion The incidence of meniscal ramp lesions was of $10.6 \%$ in ACL reconstruction surgeries, being more frequent among patients with chronic lesions. The increasing annual incidence ranged from $4.0 \%$ in 2014 to $20.6 \%$ in 2019 .

\section{Introdução}

As lesões da rampa meniscal estão comumente associadas às rupturas do ligamento cruzado anterior (LCA). Uma lesão da rampa consiste em rupturas meniscossinoviais ou meniscocapsulares, que podem ser difíceis de diagnosticar artroscopicamente a partir do compartimento anterior, e que aumentam significativamente a instabilidade anteroposterior e rotatória do joelho quando comparadas com lesões isoladas do LCA. ${ }^{1-4}$ Estudadas inicialmente por Hamberg $\left(\right.$ apud Strobel ${ }^{5}$ ), atribuiu-se pela primeira vez o termo "lesão da rampa" a essas lesões para diferenciá-las de outras lesões clássicas da porção posterior do menisco medial.

Embora exista uma longa história de reconhecimento das lesões da rampa meniscal, o tema tem sido pouco estudado nas últimas décadas. Essa falta de importância prévia atribuída ao tópico é consequência de fatores como a baixa sensibilidade da ressonância magnética (RM), com um alto número de falsos negativos, conhecimento insuficiente sobre suas consequências biomecânicas, e um senso intuitivo de que essas lesões podem se curar espontaneamente., ${ }^{3,6}$ Além disso, a lesão está localizada em um "ponto cego" do compartimento posteromedial do joelho, o que dificulta sua visualização pelos portais artroscópicos convencionais, e exige do cirurgião uma habilidade técnica mais apurada para seu acesso e reparo. ${ }^{7}$ Em 2014, Sonnery-Cottet et al. ${ }^{8}$ introduziram o conceito de "lesões ocultas", para as lesões da rampa meniscal que não são visíveis pelas abordagens artroscópicas convencionais, e propuseram uma exploração sistemática do segmento posterior do menisco medial por uma via de artroscopia posteromedial.

O diagnóstico preciso das lesões da rampa é um desafio também para os radiologistas. Em 2017, DePhillippo et al. ${ }^{9}$ publicaram um artigo que mostra uma má correlação entre a imagem e a artroscopia, com mais da metade dos casos sendo subdiagnosticados no préoperatório. Um edema na medula óssea na região posteromedial do planalto tibial medial parece ser um sinal radiológico sugestivo, presente em $72 \%$ dos casos de lesão da rampa meniscal. ${ }^{9}$

Apesar de ainda não haver dados sobre a epidemiologia das lesões da rampa na população brasileira, a literatura internacional mostra uma incidência entre $9 \%$ e $24 \%$ nas reconstruções do LCA (RLCAs). ${ }^{1,6,7,10}$ O objetivo deste estudo é avaliar a incidência e o perfil epidemiológico das lesões da rampa meniscal nos pacientes submetidos a RLCA, além de identificar possíveis fatores de risco associados a essas lesões.

\section{Materiais e Métodos}

Por meio de uma consulta aos prontuários hospitalares, fez-se uma análise retrospectiva dos dados de 824 RLCAs. O critério de inclusão foi pacientes submetidos a RLCA primária ou de revisão entre julho de 2014 e abril de 2020. Os pacientes submetidos a cirurgias concomitantes, como reconstruções multiligamentares ou osteotomias, foram excluídos. No préoperatório, todos os pacientes tiveram uma ruptura completa do LCA, diagnosticada com base no exame clínico e nos exames de RM. $O$ estudo teve a aprovação da Plataforma Brasil sob o número CAAE 27216819.2.0000.5496

\section{Técnica Cirúrgica}

Os procedimentos cirúrgicos foram realizados por dois cirurgiões especialistas em cirurgia do joelho e com grande experiência nas RLCAs. Os pacientes foram posicionados de maneira padrão para a artroscopia (-Figura 1). Lesões 


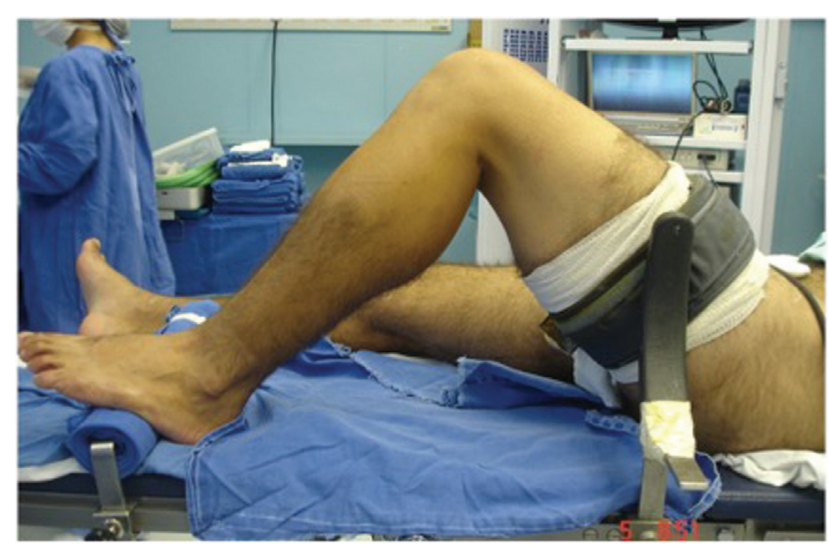

Fig. 1 Vista lateral do posicionamento do membro inferior durante a reconstrução do ligamento cruzado anterior (LCA), com o pé apoiado sobre a mesa cirúrgica, apoio lateral no nível do torniquete, e joelho com flexão de $90^{\circ}$.

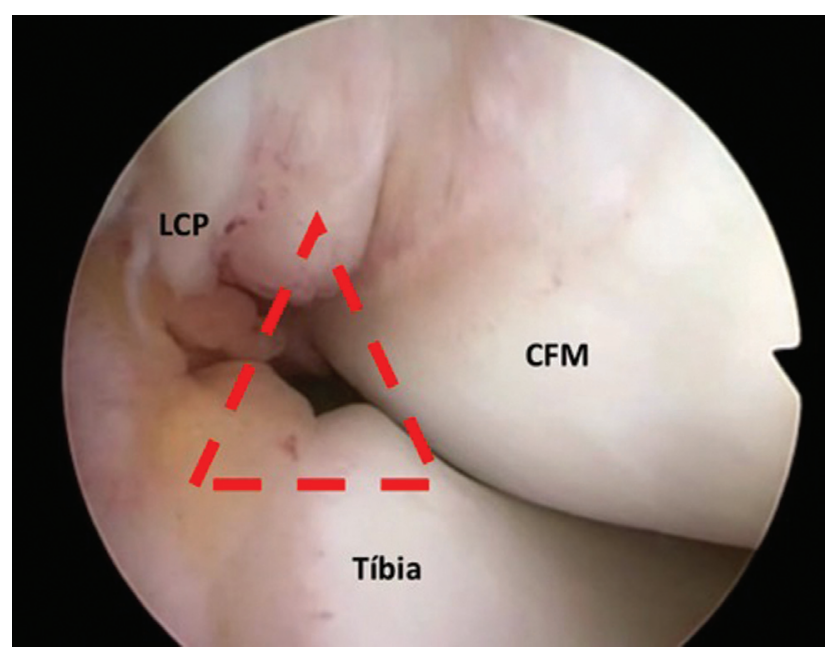

Fig. 2 Imagem de artroscopia do espaço no intercôndilo definido por onde é inserido o artroscópio para acesso ao compartimento posteromedial do joelho. O ponto correto é identificado no centro de um triângulo (em vermelho) formado pelo côndilo femoral medial (CFM), ligamento cruzado posterior (LCP), e tíbia. meniscais e/ou condrais foram abordadas antes da reconstrução ligamentar.

Inicialmente, foi realizada a exploração artroscópica dos compartimentos do joelho. Nos casos em que houve qualquer sinal de instabilidade meniscal, como aumento do deslocamento anterior sob tração, ou quando visualizou-se alguma fissura em seu folheto inferior, foi realizada uma avaliação do compartimento posteromedial do joelho, como proposto por Sonnery-Cottet et al. $^{8}$ Para essa avaliação, o artroscópio é mantido no portal anterolateral e inserido através de um espaço no intercôndilo definido pelo côndilo femoral medial, ligamento cruzado posterior (LCP), e tíbia (-Figura 2).

Nos casos em que se constatou alguma fissura do menisco (lesão da rampa), o reparo foi realizado. Quando houve, ainda, suspeita da presença de uma lesão de rampa oculta (tipo D), ${ }^{11}$ a integridade meniscal foi testada com uma agulha, e em seguida com um gancho artroscópico, inseridos através de um portal posteromedial. A confecção do portal posteromedial foi feita por meio de transiluminação, que auxilia na visualização de veia e nervo safeno que devem ser preservados (-Figura 3). A lâmina de microdebridador foi, então, inserida através do portal posteromedial, e ambas as superfícies da lesão foram cruentizadas (-Figura 4).

Para a realização das suturas, foi utilizado um gancho de sutura de $25^{\circ}$ (SutureLasso, Arthrex, Naples, FL, Estados Unidos) carregado com um fio de monofilamento absorvível $n^{\circ} 1$ (PDS, Ethicon, Inc., Somerville, NJ, Estados Unidos). A confecção dos pontos, com nós deslizantes, foi realizada com o auxílio de um empurrador de nós (- Figura 5). 0 reparo satisfatório e estável foi confirmado por avaliação com uso de um gancho artroscópico inserido pelos portais anterolateral e posteromedial. Por fim, a RLCA foi realizada com a confecção de túneis anatômicos independentes (outside-in), segundo a técnica de Chambat. ${ }^{12,13}$ Os pacientes submetidos a reparo da lesão da rampa seguiram o mesmo protocolo de reabilitação.

\section{Análise Estatística}

As variáveis quantitativas foram descritas pelas médias e pelos valores mínimo e máximo. As variáveis qualitativas

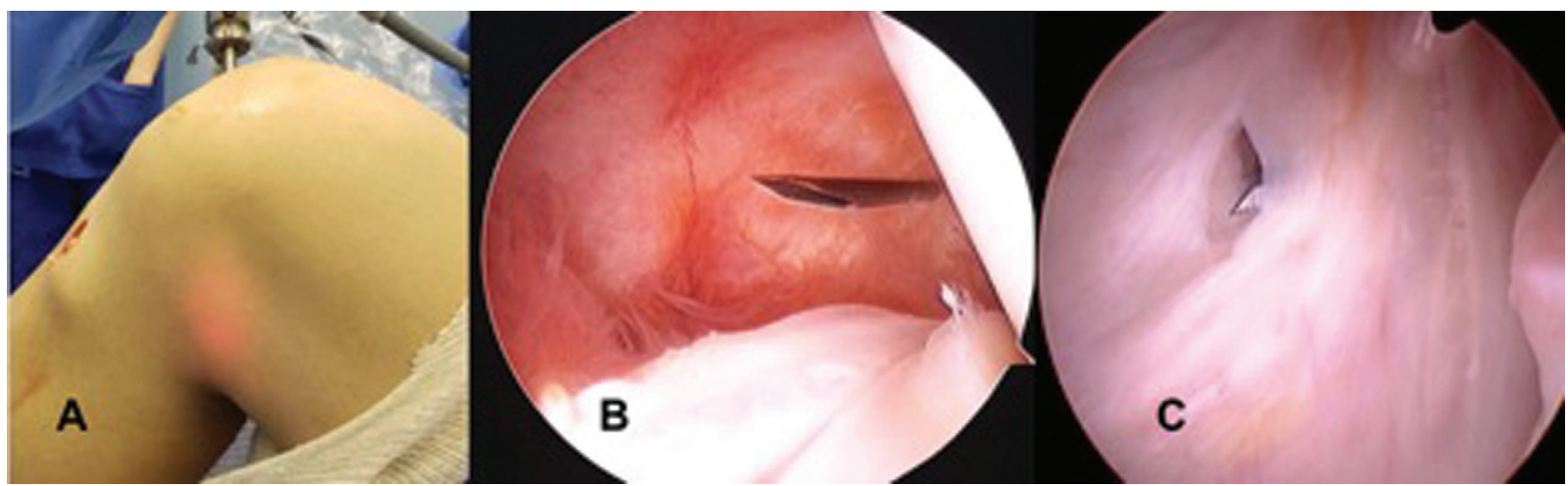

Fig. 3 Detalhes da confecção do portal posteromedial, com o artroscópio inserido no compartimento posteromedial do joelho. (A) O uso da transiluminação evita a lesão iatrogênica de vasos e nervos. (B) A agulha é introduzida na direção da lesão, para a definição do melhor ponto para criar o portal. (C) Sob visão direta, portal é criado com uso de uma lâmina de bisturi. 


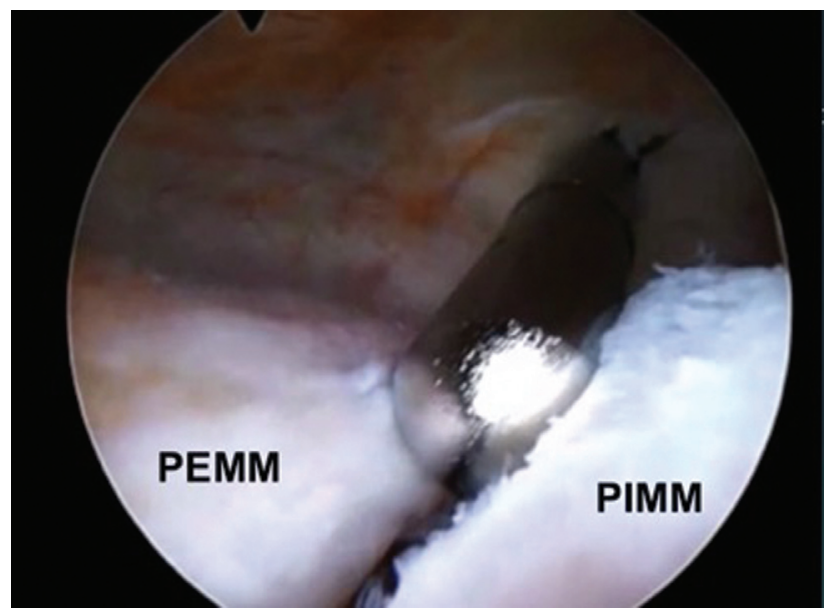

Fig. 4 Imagem da artroscopia, com o artroscópio localizado no compartimento posteromedial do joelho, evidenciando a lesão da rampa meniscal. A cruentização e regularização das bordas da lesão da rampa meniscal é realizada com uma lâmina de microdebridador. A porção externa do menisco medial (PEMM) e a porção interna do menisco medial (PIMM) são claramente visualizadas na imagem.

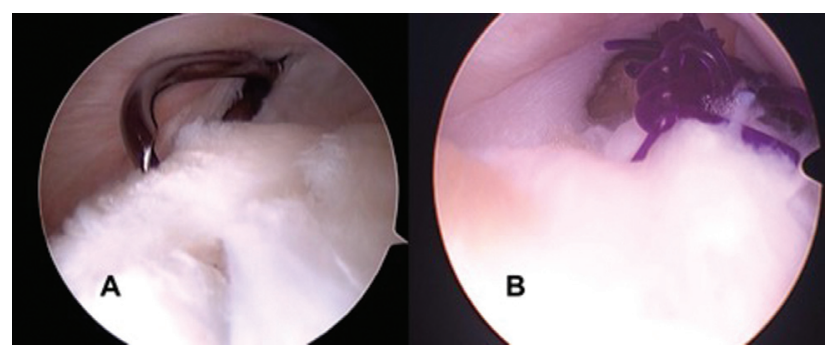

Fig. 5 Imagem artroscópica da realização da sutura no reparo da lesão da rampa através do portal posteromedial do joelho. (A) $\mathrm{O}$ gancho de sutura de $25^{\circ}$ (SutureLasso, Arthrex, Naples, FL, Estados Unidos) é introduzido pelo portal posteromedial para fazer o reparo da lesão. (B) A sutura é feita com uso de pontos simples e com o auxílio de um empurrador de nós.

foram descritas pela distribuição de frequências absoluta e relativa (\%). A análise da relação entre as variáveis qualitativas foi realizada pelo teste de associação do Qui-quadrado. As proporções de casos no total e por ano foram analisadas pelas taxas de incidência e seus respectivos intervalos de confiança de 95\% (IC95\%), e foram calculadas pela divisão entre o número de casos confirmados e o agregado da população exposta no período e expressa por 100 . O nível de significância estatística adotado foi de $5 \%$ e os dados foram analisados no programa Statistical Package for the Social Sciences (IBM SPSS Statisticis for Windows, IBM Corp., Armonk, NY, Estados Unidos), versão 24.0.

\section{Resultados}

O número total de pacientes submetidos a RLCA incluídos no estudo foi de 824. A lesão da rampa meniscal foi encontrada e reparada em $87(10,6 \%)$ pacientes. As características dos pacientes estão demonstradas na - Tabela 1.

Do total de pacientes com lesão da rampa meniscal, 76 $(87,3 \%)$ eram do sexo masculino, e $11(12,7 \%)$, do sexo
Tabela 1 Características dos pacientes submetidos a reconstrução do ligamento cruzado anterior (LCA) com e sem lesão da rampa meniscal associada

\begin{tabular}{|c|c|c|c|}
\hline Características & $\begin{array}{l}\text { Com lesão } \\
\text { - n (\%) }\end{array}$ & $\begin{array}{l}\text { Sem lesão } \\
-\mathrm{n}(\%)\end{array}$ & $\begin{array}{l}\text { Valor } \\
\text { de } p\end{array}$ \\
\hline \multicolumn{4}{|l|}{ Sexo } \\
\hline Masculino & $76(87,3 \%)$ & $631(85,6 \%)$ & \multirow[t]{2}{*}{0,718} \\
\hline Feminino & $11(12,7 \%)$ & $109(14,4 \%)$ & \\
\hline \multicolumn{4}{|l|}{ Lateralidade } \\
\hline Direita & $61(70,1 \%)$ & $403(54,7 \%)$ & \multirow[t]{2}{*}{$0,008^{*}$} \\
\hline Esquerda & $26(29,9 \%)$ & 337 (45,3\%) & \\
\hline \multicolumn{4}{|l|}{$\begin{array}{l}\text { Reconstrução } \\
\text { ligamentar }\end{array}$} \\
\hline Primária & $80(91,9 \%)$ & $694(94,2 \%)$ & \multirow[t]{2}{*}{0,562} \\
\hline Revisão & $7(8,1 \%)$ & $43(5,4 \%)$ & \\
\hline \multicolumn{4}{|l|}{ Idade (anos) } \\
\hline$<20$ & $6(6,9 \%)$ & $102(13,8 \%)$ & \multirow[t]{5}{*}{0,053} \\
\hline $20-30$ & $44(50,6 \%)$ & $267(36,2 \%)$ & \\
\hline $31-40$ & $26(29,9 \%)$ & $255(34,6 \%)$ & \\
\hline$>40$ & $11(12,6 \%)$ & $119(15,4 \%)$ & \\
\hline Média & $29,9(17-49)$ & $31,4(13-71)$ & \\
\hline \multicolumn{4}{|l|}{$\begin{array}{l}\text { Tempo de } \\
\text { lesão (meses) }\end{array}$} \\
\hline$\leq 3$ & $28(32,2 \%)$ & $362(49,1 \%)$ & \multirow[t]{6}{*}{$0,008^{*}$} \\
\hline $4-6$ & $14(16,1 \%)$ & $102(13,8 \%)$ & \\
\hline $7-12$ & $15(17,2 \%)$ & $79(10,7 \%)$ & \\
\hline $13-24$ & $14(16,1 \%)$ & $58(7,9 \%)$ & \\
\hline$>24$ & $16(18,4 \%)$ & $136(18,5 \%)$ & \\
\hline Média & $16,4(1-120)$ & $19,7(1-360)$ & \\
\hline
\end{tabular}

Nota: *Associação significativa pelo teste do Qui-quadrado para valores de $p \leq 0,05$.

feminino. A média de idade nesse grupo foi de 29,9 anos (variação: 17 a 49 anos). Das 87 RLCAs com lesão da rampa associada, $80(91,9 \%)$ eram cirurgia primária, e apenas 7 $(8,1 \%)$ eram de revisão, mas sem significância estatística entre os grupos $(p=0,562)$. Com relação à lateralidade, 0 joelho direito foi acometido em 61 casos $(70,1 \%)$, e o esquerdo, em 26 (29,9\%), o que se mostrou estatisticamente significativo $(p=0,008)$. O tempo entre a lesão e a RLCA também foi estatisticamente significativo $(p=0,008)$. Nos casos em que havia lesão da rampa, esse tempo foi de até 3 meses ( 28 casos; $32,2 \%$ ); entre 4 e 6 meses ( 14 casos; $16,1 \%$ ); entre 7 e 12 meses (15 casos; 17,2\%); de 13 a 24 meses (14 casos; $16,1 \%$ ); e acima de 24 meses (16 casos; $18,4 \%$ ), com uma média de 16,4 meses (variação: 1 a 120 meses). Já nos casos em que não foram diagnosticadas lesões da rampa, 362 (49,1\%) tinham até 3 meses de lesão; 102 (13,8\%), entre 4 e 6 meses; 79 (10,7\%), entre 7 e 12 meses; 58 (7,9\%), entre 13 e 24 meses; e 136 (18,5\%), mais de 24 meses, com uma média de 19,7 meses (variação: 1 a 360 meses). 
Tabela 2 Causas da ruptura do ligamento cruzado anterior (LCA)

\begin{tabular}{|c|c|c|c|}
\hline $\begin{array}{l}\text { Trauma não } \\
\text { relacionado } \\
\text { ao esporte }\end{array}$ & $\begin{array}{l}\text { Com lesão } \\
\text { - n (\%) }\end{array}$ & $\begin{array}{l}\text { Sem lesão } \\
-\mathrm{n}(\%)\end{array}$ & $\begin{array}{l}\text { Valor } \\
\text { dep }\end{array}$ \\
\hline Queda & $7(5,9 \%)$ & $64(54,2 \%)$ & \multirow[t]{3}{*}{0,939} \\
\hline Acidente de trânsito & $4(3,4 \%)$ & $43(36,5 \%)$ & \\
\hline Total (100\%) & $11(9,3 \%)$ & $107(90,7 \%)$ & \\
\hline $\begin{array}{l}\text { Trauma } \\
\text { relacionado } \\
\text { ao esporte }\end{array}$ & $\begin{array}{l}\text { Com lesão } \\
- \text { n (\%) }\end{array}$ & $\begin{array}{l}\text { Sem Lesão } \\
-\mathrm{n}(\%)\end{array}$ & $\begin{array}{l}\text { Valor } \\
\text { dep }\end{array}$ \\
\hline Futebol & $68(9,6 \%)$ & $548(77,6 \%)$ & \multirow[t]{5}{*}{0,599} \\
\hline Lutas & $3(0,4 \%)$ & $25(3,5 \%)$ & \\
\hline Basquete & $1(0,1 \%)$ & $14(2,0 \%)$ & \\
\hline Outros & $4(0,7 \%)$ & $43(6,1 \%)$ & \\
\hline Total (100\%) & $76(10,8 \%)$ & $630(89,2 \%)$ & \\
\hline
\end{tabular}

Tabela 3 Taxa anual de incidência de lesões da rampa

\begin{tabular}{|l|l|l|l|l|l|}
\hline Ano & $\begin{array}{l}\text { Total } \\
\text { de } \\
\text { expostos } \\
\text { (n) }\end{array}$ & $\begin{array}{l}\text { Lesões da } \\
\text { rampa (n) }\end{array}$ & $\begin{array}{l}\text { Incidência } \\
(\%)\end{array}$ & \multicolumn{2}{|l|}{ IC95\% } \\
\cline { 5 - 6 } & & & LI & LS \\
\hline 2014 & 124 & 5 & 4,00 & 1,70 & 9,10 \\
\hline 2015 & 89 & 4 & 4,50 & 1,80 & 11,00 \\
\hline 2016 & 110 & 12 & 10,90 & 6,40 & 18,10 \\
\hline 2017 & 104 & 12 & 11,50 & 6,70 & 19,10 \\
\hline 2018 & 116 & 6 & 5,20 & 2,40 & 10,80 \\
\hline 2019 & 281 & 48 & 20,60 & 15,10 & 21,90 \\
\hline Total & 824 & 87 & 10,60 & 8,60 & 12,80 \\
\hline
\end{tabular}

Abreviaturas: IC95\%, intervalo de confiança de 95\%; LI, limite inferior; LS, limite superior.

Os traumas relacionados aos esportes ou às atividades físicas foram responsáveis por $76(87,4 \%)$ casos. Entre esses, o futebol foi a causa mais frequente, com 68 casos (78,2\%; $p=0,599)$. A prática de lutas resultou em 3 casos (3,4\%); a de basquete, em 1 caso (1,1\%), e a de outros esportes, como vôlei, handebol, rúgbi e ciclismo, 4 casos (4,7\%). Entre as causas não relacionadas aos esportes (11 casos; 9,3\%), 7 (5,9\%) ocorreram devido a quedas, e $4(3,4 \%)$, a acidentes de trânsito (-Tabela 2).

A taxa de incidência anual foi ilustrada na - Tabela 3, e demonstra que, com exceção do ano de 2018 (5,2\%), houve um aumento progressivo na incidência das lesões da rampa, que variou de 4,0\%, em 2014, a 20,6\%, em 2019.

\section{Discussão}

Os principais achados do presente estudo foram a incidência das lesões da rampa meniscal em $10,6 \%$ dos pacientes com lesão do LCA, e a maior incidência nas lesões crônicas do LCA. Outros autores relataram valores entre $9 \%$ e $24 \%$. ${ }^{1,6,9,10,14-16}$ Bollen ${ }^{6}$ e Di Vico et al. ${ }^{15}$ relataramincidências menores, de 9,3\% e 9,6\%, respectivamente. Ambos os estudos incluíram uma amostra também menor, respectivamente com 183 e 115 pacientes. Liu et al., ${ }^{10}$ que contaram com uma amostra de 868 pacientes, obtiveram, por sua vez, uma incidência de $16,6 \%$, mais próxima àquela encontrada no presente estudo. Os trabalhos que encontraram as maiores incidências foram os de Seil et al. ${ }^{14}$ e de Sonnery-Cottet et al., ${ }^{1}$ ambos com $24 \%$ dos casos de lesão do LCA, analisando 224 e 3.214 pacientes, respectivamente. Essa grande variação dos resultados encontrados na literatura pode ser fruto de alguns fatores, como a experiência e a habilidade dos cirurgiões em diagnosticar a lesão durante o procedimento artroscópico, e o número de pacientes incluídos na amostra. Ficou evidente, no presente estudo, que houve um importante aumento anual na incidência das lesões da rampa, de 4\% a 20,6\% entre 2014 e 2019. Atribui-se isso ao fato de que, quanto mais familiarizado e experiente for o cirurgião em investigar e reparar essa lesão, maior será o número de diagnósticos. ${ }^{17}$

O acompanhamento de pacientes submetidos a RLCA, com menisco medial inicialmente considerado saudável, mostrou que alguns pacientes ainda degradam o menisco medial, apesar de o joelho parecer clinicamente estável. ${ }^{18}$ Isso demonstra que uma parte das lesões é subdiagnosticada.

Em relação aos fatores de risco, é sabido que o tempo entre a lesão e a RLCA está associado a uma incidência crescente das lesões do menisco medial. ${ }^{1,19,20}$ Church e Keating ${ }^{21}$ encontraram um aumento no número de todos os tipos de lesões meniscais após doze meses, e recomendaram RLCA precoce para evitar essas lesões. Liu et al. ${ }^{10}$ mostraram que, em até 24 meses entre a lesão do LCA e a cirurgia, houve um aumento da incidência de lesões da rampa. No presente estudo, identificou-se que, dos casos em que esse tempo foi menor do que 3 meses, 32,2\% tinham a lesão da rampa meniscal, enquanto 49,1\% não apresentavam sinais de lesão. Nos casos crônicos, com tempo maior do que 3 meses, houve um aumento proporcional de $67,8 \%$ nos 59 casos em que havia lesão da rampa, em comparação com 50,9\%, nos 375 casos em que o menisco estava íntegro ( $p$ 0,008).

Em relação aos casos de revisão, a literatura ${ }^{22-24}$ mostra que joelhos submetidos a revisão do LCA têm mais lesões intra-articulares do que joelhos submetidos a reconstrução primária. Wright et al. ${ }^{25}$ mostraram que até $90 \%$ dos casos de revisão do LCA apresentaram uma lesão meniscal ou condral, enquanto $57 \%$ tinham ambas as lesões no momento da cirurgia. Assim como Sonnery-Cottet et al., ${ }^{1}$ optamos por incluir no estudo os pacientes submetidos a revisão do LCA, buscando avaliar se isso também pode ser considerado um fator de risco. Curiosamente, os dados encontrados no presente estudo não mostraram maior incidência quando se tratava, especificamente, das lesões da rampa meniscal nos casos de revisão $(p=0,562)$.

Apesar da incidência de 10,6\% das RLCAs, a incidência anual no período estudado variou de $4,0 \%$, em 2014 , a $20,6 \%$, em 2019. Essa escalada no número de casos chama a atenção, e pode demonstrar uma significativa curva de aprendizado no diagnóstico e no tratamento dessas lesões. Portanto, recomenda-se a criação de uma rotina de inspeção sistemática do compartimento posteromedial do joelho nas RLCAs, principalmente nos casos crônicos. 
Este estudo apresenta algumas limitações. Durante os primeiros anos, alguns casos podem ter sido subdiagnosticados em decorrência da curva de aprendizado, e podem ter apresentado uma incidência um pouco abaixo da real. Além disso, apesar de ambos os cirurgiões terem vasta experiência nas cirurgias de RLCA, eles não necessariamente estavam no mesmo ponto da curva de aprendizado para o diagnóstico e o reparo das lesões da rampa meniscal durante o período estudado. Isso pode ter influenciado o resultado final e a incidência anual. Outro possível viés é o fato de ambos os cirurgiões serem destros, o que pode justificar o maior número (estatisticamente significativo) da lateralidade direita, talvez por uma facilidade técnica e preferência dos cirurgiões nos primeiros casos. O presente estudo também tem pontos fortes, como a amostra significativa (824 pacientes) em comparação com as de outros estudos epidemiológicos semelhantes na literatura. $\mathrm{O}$ fato de o estudo envolver mais de um serviço fortalece o artigo, que analisa a epidemiologia dessas lesões sob o mesmo protocolo de exploração artroscópica, porém, em regiões distintas do território nacional. De qualquer maneira, novos estudos epidemiológicos devem ser realizados nos diferentes centros e regiões brasileiras. Com um maior número de informações e dados de diferentes cirurgiões de joelho, haverá uma melhor compreensão dos fatores de risco e da incidência das lesões da rampa meniscal na população brasileira.

\section{Conclusão}

A incidência das lesões da rampa meniscal foi de $10,6 \%$ nas RLCAs, sendo mais frequente nas lesões crônicas. A incidência anual no período estudado variou de 4,0\%, em 2014, a 20,6\%, em 2019.

\section{Suporte Financeiro}

Não houve suporte financeiro de fontes públicas, comerciais, ou sem fins lucrativos.

\section{Conflito de Interesses}

Os autores declaram não haver conflito de interesses.

\section{Referências}

1 Sonnery-Cottet B, Praz C, Rosenstiel N, et al. Epidemiological Evaluation of Meniscal Ramp Lesions in 3214 Anterior Cruciate Ligament-Injured Knees From the SANTI Study Group Database: A Risk Factor Analysis and Study of Secondary Meniscectomy Rates Following 769 Ramp Repairs. Am J Sports Med 2018;46(13): 3189-3197

2 Mouton C, Magosch A, Pape D, Hoffmann A, Nührenbörger C, Seil R. Ramp lesions of the medial meniscus are associated with a higher grade of dynamic rotatory laxity in ACL-injured patients in comparison to patients with an isolated injury. Knee Surg Sports Traumatol Arthrosc 2020;28(04):1023-1028

3 Sonnery-Cottet B, Serra Cruz R, Vieira TD, Goes RA, Saithna A. Ramp Lesions: An Unrecognized Posteromedial Instability? Clin Sports Med 2020;39(01):69-81
4 Ahn JH, Bae TS, Kang KS, Kang SY, Lee SH. Longitudinal tear of the medial meniscus posterior horn in the anterior cruciate ligamentdeficient knee significantly influences anterior stability. Am J Sports Med 2011;39(10):2187-2193

5 Strobel MJ. Menisci. In: Fett HM, Flechtner P, eds. Manual of Arthroscopy Surgery. New York: Springer; 1988:171-178

6 Bollen SR. Posteromedial meniscocapsular injury associated with rupture of the anterior cruciate ligament: a previously unrecognised association. J Bone Joint Surg Br 2010;92(02):222-223

7 Chahla J, Dean CS, Moatshe G, et al. Meniscal Ramp Lesions: Anatomy, Incidence, Diagnosis, and Treatment. Orthop J Sports Med 2016;4(07):2325967116657815

8 Sonnery-Cottet B, Conteduca J, Thaunat M, Gunepin FX, Seil R. Hidden lesions of the posterior horn of the medial meniscus: a systematic arthroscopic exploration of the concealed portion of the knee. Am J Sports Med 2014;42(04):921-926

9 DePhillipo NN, Cinque ME, Chahla J, Geeslin AG, Engebretsen L, LaPrade RF. Incidence and Detection of Meniscal Ramp Lesions on Magnetic Resonance Imaging in Patients With Anterior Cruciate Ligament Reconstruction. Am J Sports Med 2017;45(10): 2233-2237

10 Liu X, Feng H, Zhang H, Hong L, Wang XS, Zhang J. Arthroscopic prevalence of ramp lesion in 868 patients with anterior cruciate ligament injury. Am J Sports Med 2011;39(04):832-837

11 Thaunat M, Fayard JM, Guimaraes TM, Jan N, Murphy CG, SonneryCottet B. Classification and Surgical Repair of Ramp Lesions of the Medial Meniscus. Arthrosc Tech 2016;5(04):e871-e875

12 Garofalo R, Mouhsine E, Chambat P, Siegrist O. Anatomic anterior cruciate ligament reconstruction: the two-incision technique. Knee Surg Sports Traumatol Arthrosc 2006;14(06):510-516

13 de Pádua VB, Maldonado H, Vilela JC, Provenza AR, Monteiro C, de Oliveira Neto HC. Comparative study of ACL reconstruction with anatomical positioning of the tunnels using the patellar tendon versus hamstrings tendon. Rev Bras Ortop 2015;47(01):50-56

14 Seil R, Mouton C, Coquay J, et al. Ramp lesions associated with ACL injuries are more likely to be present in contact injuries and complete ACL tears. Knee Surg Sports Traumatol Arthrosc 2018; 26(04):1080-1085

15 Di Vico G, Di Donato SL, Balato G, et al. Correlation between time from injury to surgery and the prevalence of ramp and hidden lesions during anterior cruciate ligament reconstruction. A new diagnostic algorithm. Muscles Ligaments Tendons J 2018;7(03): 491-497

16 Hatayama K, Terauchi M, Saito K, Aoki J, Nonaka S, Higuchi H. Magnetic Resonance Imaging Diagnosis of Medial Meniscal Ramp Lesions in Patients With Anterior Cruciate Ligament Injuries. Arthroscopy 2018;34(05):1631-1637

17 Sonnery-Cottet B. Editorial Commentary: Ramp Lesion: The Eye Sees Only What the Mind Is Prepared to Comprehend. Arthroscopy 2020;36(11):2934-2937

18 Rochcongar G, Cucurulo T, Ameline T, et al. Meniscal survival rate after anterior cruciate ligament reconstruction. Orthop Traumatol Surg Res 2015;101(8, Suppl)S323-S326

19 Woods GW, Chapman DR. Repairable posterior menisco-capsular disruption in anterior cruciate ligament injuries. Am J Sports Med 1984;12(05):381-385

20 Kennedy J, Jackson MP, O'Kelly P, Moran R. Timing of reconstruction of the anterior cruciate ligament in athletes and the incidence of secondary pathology within the knee. J Bone Joint Surg $\mathrm{Br}$ 2010;92(03):362-366

21 Church S, Keating JF. Reconstruction of the anterior cruciate ligament: timing of surgery and the incidence of meniscal tears and degenerative change. J Bone Joint Surg Br 2005;87(12): 1639-1642 
22 Drogset JO, Grøntvedt T. Anterior cruciate ligament reconstruction with and without a ligament augmentation device : results at 8-Year follow-up. Am J Sports Med 2002;30(06):851-856

23 George MS, Dunn WR, Spindler KP. Current concepts review: revision anterior cruciate ligament reconstruction. Am J Sports Med 2006;34(12):2026-2037

24 Brophy RH, Haas AK, Huston LJ, Nwosu SK; Wright RWMARS Group. Association of Meniscal Status, Lower Extremity Align- ment, and Body Mass Index With Chondrosis at Revision Anterior Cruciate Ligament Reconstruction. Am J Sports Med 2015;43(07): 1616-1622

25 Wright RW, Huston LJ, Spindler KP, et al. Descriptive epidemiology of the Multicenter ACL Revision Study (MARS) cohort. Am J Sports Med 2010;38(10):1979-1986 